
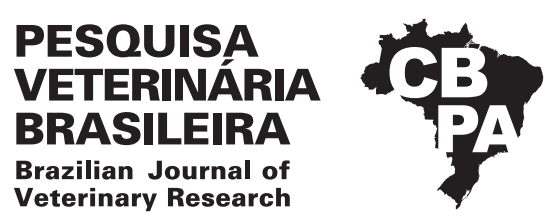

Pesq. Vet. Bras. 38(7):1307-1312, julho 2018 DOI: $10.1590 / 1678-5150-P V B-5531$

Artigo Original

ISSN 0100-736X (Print)

ISSN 1678-5150 (Online)

\title{
Sazonalidade da mosca-dos-chifres, Haematobia irritans, no semiárido brasileiro ${ }^{1}$
}

\author{
Márcia A. Medeiros ${ }^{2}$, Antonio Thadeu M. Barros ${ }^{3}$, Rosane Maria T. Medeiros ${ }^{4}$, \\ Vanessa D. Vieira ${ }^{2}$, Sérgio S. Azevedo ${ }^{2}$ e Franklin Riet-Correa ${ }^{4 *}$
}

\begin{abstract}
Medeiros M.A., Barros A.T.M., Medeiros R.M.T., Vieira V.D., Azevedo S.S. \& Riet-Correa F. 2018. [Seasonality of horn flies (Haematobia irritans) in the Brazilian semi-arid.] Sazonalidade da mosca-dos-chifres, Haematobia irritans, no semiárido paraibano. Pesquisa Veterinária Brasileira 38(7):1307-1312. Hospital Veterinário, Centro de Saúde e Tecnologia Rural, Universidade Federal de Campina Grande, Campus de Patos, PB 58708-110, Brazil. E-mail: frcorrea@inia.org.uy

The horn fly (Haematobia irritans) is a major livestock pest in Brazil. Despite of the regional needs, no studies assessing its importance and subsidizing its control are available for the Brazilian northeast. The present study aimed to know the population dynamics and determine the infestation levels of $H$. irritans on cattle in the semiarid region of the Paraíba state, in order to support control programs. The study was conducted at the Nucleus of Research for the Semiarid Tropics, Federal University of Campina Grande, municipality of Patos, Paraíba. During 42 months, from April 2011 to September 2014, horn fly counts were performed every 14 days in a Sindi herd ( 26 cows and a bull). The main climatic parameters were recorded daily. Horn fly infestations occurred throughout the study, with individual infestations above 100 flies at all counts, monthly mean infestations of up to 183 flies/cow and maximum individual infestations of 500 flies on cows and 1,800 flies on the bull. Influenced by microclimatic influence of large reservoirs, the horn fly population showed a bimodal behavior, with population peaks in October/November and March. No significant correlation between climatic factors and fly counts was found. In order to avoid infestation peaks and to reduce subsequent economic losses, control measures are recommended.
\end{abstract}

INDEX TERMS: Seasonality, horn flies, Haematobia irritans, Brazilian semi-arid, ectoparasite, population dynamics, strategic control.

RESUMO-- A mosca-dos-chifres (Haematobia irritans) é uma dos principais pragas que afetam a pecuária no Brasil. A despeito das carências regionais, inexistem estudos que avaliem sua importância e subsidiem seu controle no nordeste brasileiro. 0 presente estudo objetivou conhecer a dinâmica populacional e determinar os níveis de infestação da H. irritans no semiárido paraibano, visando subsidiar

\footnotetext{
${ }^{1}$ Recebido em 7 de setembro de 2017.

Aceito para publicação em 14 de setembro de 2017.

Parte da Tese de Doutorado do primeiro autor, Programa de Pós-Graduação em Medicina Veterinária.

${ }^{2}$ Hospital Veterinário, Centro de Saúde e Tecnologia Rural (CSTR), Universidade Federal de Campina Grande (UFCG), Av. Universitária s/n, Santa Cecília, Patos, PB 58708-110, Brasil.

${ }^{3}$ Empresa Brasileira de Pesquisa Agropecuária (Embrapa), Centro Nacional de Pesquisa de Gado de Corte, Campo Grande, MS 79106-550, Brasil.

${ }^{4}$ Instituto Nacional de Investigación Agropecuaria (INIA), La Estanzuela, Colonia, Uruguay. *Autor para correspondência: frcorrea@inia.org.uy
}

programas de controle. 0 estudo foi conduzido no Núcleo de Pesquisa para o Trópico Semiárido, Universidade Federal de Campina Grande, município de Patos, Paraíba. Durante 42 meses, de abril 2011 a setembro 2014, contagens de moscas-dos-chifres foram realizadas a cada 14 dias em um rebanho Sindi (26 vacas e um touro). Paralelamente, foram registrados diariamente os principais parâmetros climáticos. Infestações da mosca-dos-chifres ocorreram durante todo o período de estudo, com infestações individuais superiores a 100 moscas em todas as contagens, infestações médias mensais de até 183 moscas/vaca e infestações individuais máximas de 500 moscas nas vacas e 1.800 moscas no touro. Influenciados pela influência microclimática de grandes açudes, a mosca-dos-chifres apresentou comportamento bimodal, com picos populacionais anuais em outubro/novembro e março. Não foi encontrada correlação significativa entre fatores climáticos e resultados das contagens. De modo a 
evitar os picos de infestação e reduzir perdas econômicas subsequentes, medidas de controle são recomendadas.

TERMOS DE INDEXAÇÃO: Sazonalidade, mosca-dos-chifres, Haematobia irritans, semiárido Paraibano, ectoparasito, dinâmica populacional, controle estratégico.

\section{INTRODUÇÃO}

Com reconhecida importância em vários países, a mosca-doschifres (Haematobia irritans) é um dos principais ectoparasitos que afetam a bovinocultura nacional (Grisi et al. 2014). Perdas econômicas devido às infestações são resultantes do incômodo causado pelas frequentes picadas, acarretando em maior gasto energético e redução do tempo de pastejo, o que leva a um atraso no ganho de peso e maior idade ao abate (Bianchin \& Alves 2002).

0 controle da mosca-dos-chifres baseia-se fundamentalmente na aplicação de produtos inseticidas sintéticos. Frequentemente realizado de forma abusiva e pouco criteriosa, o controle químico da mosca tem contribuído para o aumento dos custos de produção e levado ao desenvolvimento de resistência (Barros et al. 2012). Assim, o conhecimento sobre a epidemiologia da espécie, assim como o uso adequado das alternativas disponíveis, torna-se essencial ao correto direcionamento das ações de controle.

A sazonalidade da mosca-dos-chifres é influenciada pelas condições climáticas locais. Em regiões de clima temperado, a ocorrência das infestações é dependente da temperatura, ao passo que, sem as limitações do inverno, infestações ocorrem praticamente durante todo o ano nas regiões tropicais; por outro lado, a abundância da mosca é influenciada pelas chuvas e umidade (Gordon et al. 1984, Barros 2001, Lima et al. 2003). Por suas características bióticas e abióticas, o semiárido brasileiro é uma região peculiar, para a qual inexistem informações epidemiológicas disponíveis sobre este parasito.

Embora estudos ecológicos sejam essenciais a um adequado controle parasitário, a carência de informações específicas para o semiárido limita as tomadas de decisão do produtor e a definição de estratégias e programas locais de controle da mosca-dos-chifres. 0 presente estudo objetivou conhecer a dinâmica populacional e os níveis de infestação da $H$. irritans em bovinos no semiárido paraibano, visando subsidiar recomendações de controle deste parasito na região.

\section{MATERIAL E MÉTODOS}

O estudo foi realizado de abril 2011 a setembro 2014 no Núcleo de Pesquisa para o Trópico Semiárido ("Fazenda Nupeárido"), da Universidade Federal de Campina Grande (07을 $\left.25^{\prime \prime S}, 37^{\circ} 16^{\prime} 41^{\prime \prime} 0\right)$, município de Patos, PB. Localizada a uma altitude de $242 \mathrm{~m}$, a fazenda situa-se às margens do Açude do Jatobá e aproximadamente a 2,9 km do Açude da Farinha (Fig.1), grandes reservatórios que abastecem a região, com capacidade para 17 e 27 milhões de metros cúbicos de água, respectivamente.

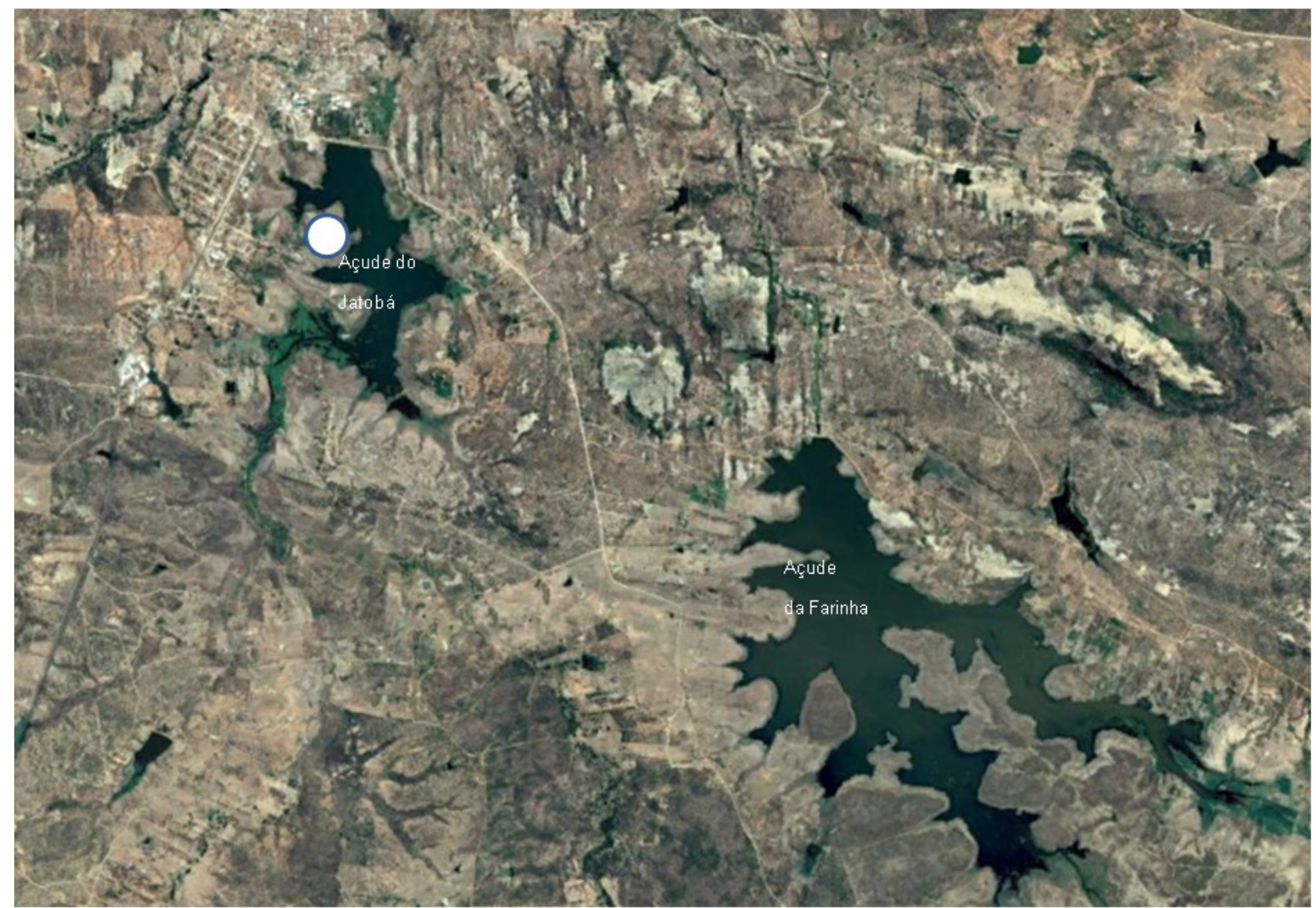

Fig.1. Localização do Núcleo de Pesquisa para o Trópico Semiárido, Universidade Federal de Campina Grande (marcado no círculo branco), às margens do Açude do Jatobá, município de Patos, PB. 
A região apresenta clima semiárido quente - Bsh, segundo a Classificação climática de Köppen-Geiger, com índices pluviométricos anuais de 600 a $700 \mathrm{~mm}$, estação chuvosa de janeiro a maio (>90\% das chuvas) e estação seca de junho a dezembro. A temperatura média anual é de $30,6^{\circ} \mathrm{C}$, com amplitude de 28,7 a $32,5^{\circ} \mathrm{C}$ e pouca variação ao longo do ano (INMET 1993). Durante o período de estudo, as variáveis climáticas temperatura, precipitação pluviométrica e umidade relativa do ar foram registradas diariamente em estação meteorológica (Estação INMET 82791, Patos, PB) a cerca de 8 km da área de estudo.

Na época do estudo, a fazenda apresentava um total de 62 animais. Por questões de manejo, as infestações da mosca-dos-chifres foram monitoradas em 26 vacas e um touro que permanecia junto às vacas. No início do experimento as 26 vacas estavam em lactação e eram ordenhadas diariamente. Os animais tinham idade entre quatro a 10 anos de idade e foram mantidos sem tratamento antiparasitário durante todo o estudo em um sistema semiextensivo em uma área de campo nativo com predominância de malva-branca (Sida sp.) e capim panasco (Aristida sp.). Diariamente, no final da tarde, o rebanho experimental era recolhido a um piquete próximo ao curral e as vacas eram ordenhadas. Durante o período de escassez de forragem, as 26 vacas do experimento e o touro foram mantidas em campo nativo e suplementadas com capim elefante (Pennisetum ssp.) picado e farelo de milho. Durante o experimento nove vacas foram tiradas do rebanho e substituídas por vacas em lactação.

Contagens individuais do número de moscas foram realizadas a cada 14 dias em todos os animais do rebanho. As contagens foram efetuadas por um único observador, entre 7:00 e 8:30h da manhã, nos animais imobilizados no brete. Infestações totais foram estimadas com base no número de moscas contado em um dos lados do animal (Morrison \& Foil 1995, Bianchin \& Alves 2002). Contagens individuais de moscas foram realizadas em infestações até 30 moscas, sendo o número de moscas estimado, a partir de grupos de 5, 10 ou 50 moscas, em infestações maiores.

Uma vez que infestações nos touros foram, de modo geral, muito superiores às das vacas, apenas os resultados das infestações nas vacas foram considerados nas análises. Foi realizada análise descritiva e de correlação (coeficiente de correlação de Pearson), através do programa Statistical Analysis System (SAS Institute 1989), entre o número médio de moscas/animal e parâmetros climáticos (temperatura média, umidade relativa média e precipitação pluviométrica acumulada) nas datas de contagem. Comparação das contagens do número de moscas entre meses e entre anos foi realizada por meio da análise de variância com um fator (ANOVA one-way).

\section{RESULTADOS}

De modo geral, as condições climáticas observadas durante o estudo foram típicas para a região, com temperaturas médias mensais variando de 24,5 a $31^{\circ} \mathrm{C}$ e umidade relativa entre $49 \%$ e $75 \%$ (média de $57,7 \%$ ). Para uma região com índice pluviométrico anual de 600-700 mm, os anos de 2011 (911,7mm) e 2014 (779,8mm) foram chuvosos, enquanto 2012 $(219,6 \mathrm{~mm})$ e $2013(427 \mathrm{~mm})$ foram particularmente secos. 0 período de janeiro a abril foi o mais chuvoso, enquanto o bimestre agosto-setembro foi o mais seco; sem chuvas em 2011 e 2013 e com apenas 1,7mm em 2014, setembro foi o mês mais seco durante o estudo (Fig.2).
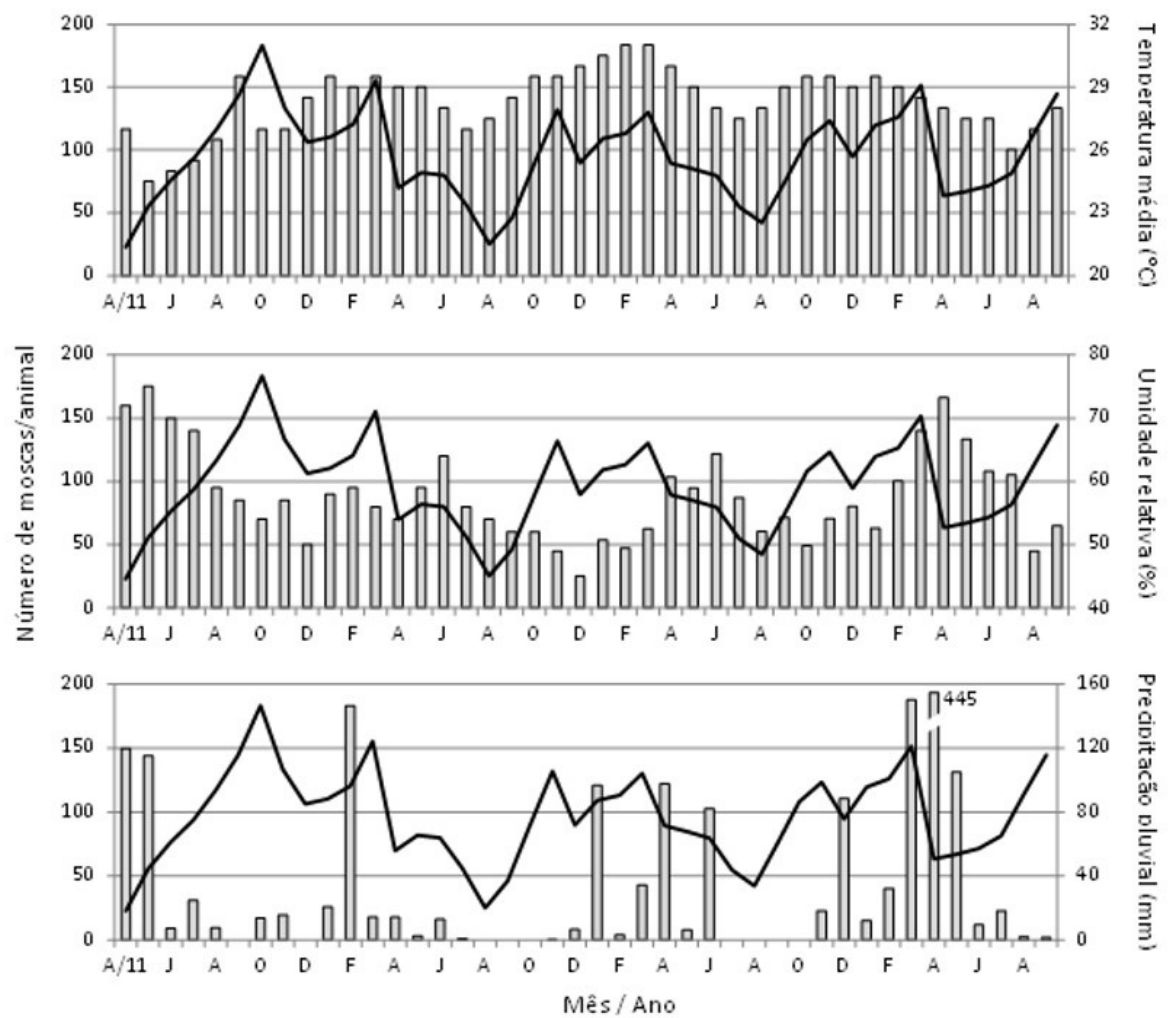

Fig.2. Sazonalidade da mosca-dos-chifres e relação com variáveis climáticas no semiárido paraibano, município de Patos, de abril 2011 a setembro 2014. 
Infestações da mosca-dos-chifres foram observadas durante todo o período de estudo, em pelo menos $96 \%$ dos animais do rebanho. Infestações superiores a 100 moscas/animal foram observadas em todas as contagens, em até $63 \%$ dos animais do rebanho (Quadro 1). Infestações médias atingiram 264 moscas/animal (outubro 2011), sendo 500 e 1.800 moscas as maiores infestações individuais nas vacas e no touro, respectivamente. A baixa infestação observada em abril 2011 (24,8 moscas/animal) foi provavelmente decorrente de tratamentos inseticidas anteriores ao início do estudo.

A mosca-dos-chifres apresentou comportamento bimodal, com picos populacionais em outubro/novembro e março (Fig.2 e 3) e menores infestações geralmente em agosto. Um rápido e acentuado aumento das infestações foi observado nos meses que antecederam o pico populacional de outubro/novembro e um progressivo declínio ocorreu após o pico de março (Fig.3).

Resultados das contagens de moscas no rebanho não apresentaram diferenças estatisticamente significativas entre os anos de estudo; entretanto, as contagens diferiram significativamente $(\mathrm{P}<0,05)$ entre os meses de pico populacional (outubro, novembro e março) e os de menores infestações (maio-julho). Não foi observada correlação significativa entre as contagens de moscas e variáveis climáticas.

\section{DISCUSSÃO}

Infestações da mosca-dos-chifres relativamente baixas, com médias inferiores a 85 moscas/animal, têm sido frequentemente observadas em estudos realizados no país, com animais zebuínos ou cruzados (Barros 2001, Bianchin \& Alves 2002, Lima et al. 2003); mesmo em animais de raças europeias, infestações médias raramente excederam 100 moscas/animal (Souza et al. 2005). No presente estudo, realizado com animais de raça zebuína (Sindi), infestações médias superiores a 100 moscas/animal foram observadas em 45,2\% das contagens efetuadas. É possível que a utilização de animais mais velhos, entre 4 a 10 anos, tenha contribuído para as maiores infestações observado neste estudo, uma vez que o nível da infestação é influenciado pela idade (Christensen \& Dobson 1979). Em gado Nelore, Bianchin et al. (2004) observaram infestações médias 3,5x e 9,3x maiores em machos de dois e três anos de idade, respectivamente, em comparação a animais de um ano.

A dinâmica populacional da mosca-dos-chifres é influenciada pelas condições climáticas locais, com marcantes diferenças geográficas. De modo geral, a temperatura determina a presença ou ausência da mosca, enquanto chuvas e umidade determinam sua abundância (Gordon et al. 1984). O rigor do inverno torna evidente a influência da temperatura em regiões temperadas (Guglielmone et al. 1997, Cruz-Vázquez et al. 2000, Castro et al. 2008), enquanto em regiões tropicais as infestações tendem a ocorrer ao longo de todo o ano e as flutuações de abundância estão frequentemente associadas ao padrão de chuvas (Collares 1990, Oliveira \& Freitas 1997, Barros 2001, Lima et al. 2003, Bianchin et al. 2006).

Na região semiárida, a pouca variação de temperatura $\left(24,5-31,0^{\circ} \mathrm{C}\right)$ observada ao longo do estudo explica a ocorrência de infestações da mosca-dos-chifres durante todo

Quadro 1. Frequência (\%) mensal de bovinos com infestações acima de 100 moscas em rebanho Sindi, de abril 2011 a setembro 2014, no semiárido paraibano

\begin{tabular}{|c|c|c|c|c|c|c|c|c|c|c|c|c|}
\hline \multirow{2}{*}{ Ano } & \multicolumn{12}{|c|}{ Mês } \\
\hline & $\mathrm{J}$ & $\mathrm{F}$ & $M$ & $\mathrm{~A}$ & $M$ & $\mathrm{~J}$ & $\mathrm{~J}$ & $\mathrm{~A}$ & $S$ & 0 & $\mathrm{~N}$ & $\mathrm{D}$ \\
\hline 2011 & . & - & - & 11,1 & 18,5 & 22,2 & 25,9 & 33,3 & 33,3 & 37,0 & 44,4 & 37,0 \\
\hline 2012 & 55,6 & 40,7 & 44,4 & 44,4 & 40,7 & 51,9 & 29,6 & 3,7 & 14,8 & 55,6 & 33,3 & 40,7 \\
\hline 2013 & 51,9 & 48,1 & 51,9 & 37,0 & 51,9 & 44,4 & 18,5 & 7,4 & 40,7 & 63,0 & 33,3 & 40,7 \\
\hline 2014 & 63,0 & 51,9 & 48,1 & 29,6 & 29,6 & 29,6 & 37,0 & 48,1 & 51,9 & - & - & - \\
\hline
\end{tabular}

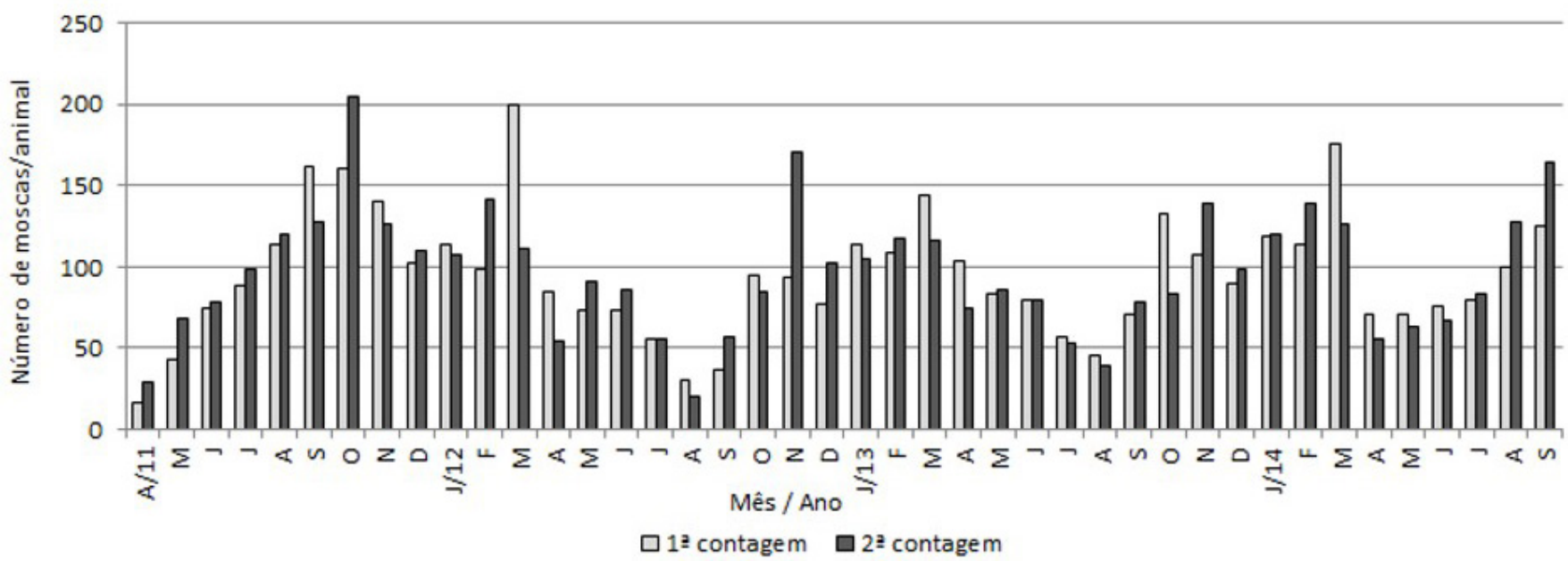

Fig.3. Infestações médias bimensais da mosca-dos-chifres em bovinos Sindi no semiárido paraibano, município de Patos, de abril 2011 a setembro 2014. 
o período. Por outro lado, dois picos populacionais anuais foram observados no semiárido paraibano, sendo o primeiro após o início das chuvas (março) e o segundo na época seca (outubro/novembro). Estes picos, observados durante os três anos e meio do estudo, denotam um consistente comportamento bimodal da mosca na região, semelhante ao observado em outras regiões do país (Alves-Branco et al. 1997, Barros 2001, Lima et al. 2003, Bianchin et al. 2006).

Os picos populacionais ocorridos em março no semiárido estiveram associados ao início da época chuvosa e coincidem com os picos observados entre outubro e dezembro em outras regiões brasileiras. Contudo, o aumento da infestação na época seca do semiárido (outubro/novembro) distingue-se do padrão conhecido e requer uma abordagem mais detalhada.

Apesar da baixa umidade característica da região semiárida, a umidade média durante os picos de infestação ocorridos na seca $(52,4 \%)$ foi próxima da registrada nos picos da estação chuvosa (58,8\%); assim como a temperatura média, nos meses de pico na seca $\left(28,7^{\circ} \mathrm{C}\right)$ e na época chuvosa $\left(29,7^{\circ} \mathrm{C}\right)$. A presença de reservatórios (como os açudes do Jatobá e da Farinha) afeta o clima em seu entorno, sendo esta influência mais importante quanto mais seco o ambiente ao redor (Dias et al. 1999). Tal influência microclimática é proporcional à proximidade do espelho d'água e inclui o aumento da umidade relativa e diminuição da temperatura, tanto no período seco como no chuvoso, como verificado na região semiárida cearense em decorrência do Açude "Castanhão" (Santos Júnior \& Caracristi 2012). Uma vez que os registros climáticos analisados no presente estudo foram obtidos a cerca de $8 \mathrm{~km}$ da área experimental, é provável que temperaturas mais amenas e umidade mais elevada tenham ocorrido no local do estudo, localizado às margens do Açude do Jatobá e a menos de $3 \mathrm{~km}$ do Açude da Farinha, favorecendo o desenvolvimento da mosca mesmo em períodos críticos de chuvas na região. Em última análise, a dinâmica populacional da mosca refletiu o microclima local, podendo diferir em situações sem a influência de grandes reservatórios.

Embora escassas, chuvas ocorreram nos períodos de seca. Coincidindo com os picos populacionais da mosca, chuvas foram registradas em outubro 2011 (13,6mm) após estiagem no mês anterior, em novembro $2012(0,2 \mathrm{~mm})$ após ausência de chuvas por três meses e em novembro 2013 (18,2mm) após quatro meses sem chuva. Mesmo durante a seca, as condições climáticas locais permitiram um aumento da abundância da mosca, culminando com a ocorrência de picos populacionais, aparentemente associados a qualquer nível de precipitação pluvial após um período de estiagem (Fig.2). Vale ressaltar que a ocorrência de elevadas infestações no período seco, época de restrição de forragem, estressa duplamente o gado (Gordon et al. 1984), situação evidenciada no presente estudo.

Não foi encontrada correlação significativa entre as infestações nos animais e os fatores climáticos analisados, o que pode ser explicado pela influência do microclima na área do estudo e grandes variações no número de moscas (picos de abundância) em situações climaticamente distintas (estações seca e chuvosa).

Nos períodos de maior abundância, os picos de infestação apresentaram curta duração, com redução do número de moscas no rebanho em uma ou duas semanas (Fig.3), situação semelhante à encontrada por Barros (2001) no Pantanal sul-mato-grossense. Contudo, níveis de infestação menores que os observados no presente estudo, principalmente no intervalo entre picos populacionais (outubro a março), têm sido previamente associados a significativas perdas à produção (Maciel et al. 2015, Bianchin et al. 2004), evidenciando um potencial impacto econômico da mosca-dos-chifres nas condições estudadas e a necessidade de seu controle na região.

As informações obtidas sobre a sazonalidade da mosca permitem o estabelecimento de um calendário de controle estratégico para a região. Neste contexto, tratamentos inseticidas no início de outubro e final de fevereiro podem evitar os picos de infestação e reduzir prejuízos econômicos subsequentes. 0 tratamento estratégico de outubro pode ser realizado em novembro, aproveitando a vacinação contra Febre Aftosa, caso as infestações se mantenham em níveis considerados aceitáveis em outubro. Tratamentos táticos podem ser eventualmente necessários em situações de elevadas infestações, traduzidas por uma intensa inquietação do gado.

\section{CONCLUSÕES}

O semiárido paraibano oferece condições para o desenvolvimento da mosca-dos-chifres durante todo o ano. As infestações apresentam um padrão bimodal, com picos populacionais anuais em março (após início das chuvas) e outubro/novembro (época seca).

Os picos de infestação nas estações chuvosas se assemelham à dinâmica da mosca em outras regiões do país. No entanto, os picos de infestação na seca estão aparentemente associados a um microclima favorável (em função de grandes açudes locais) e ocorrência de chuva após um período de estiagem.

A dinâmica populacional da mosca-dos-chifres adquire características peculiares no semiárido, uma vez que os principais fatores climáticos em outras regiões apresentam pouca variação (temperatura) ou frequência escassa e irregular (chuvas), aliada à influência microclimática de grandes reservatórios, relativamente comuns no nordeste.

Agradecimentos.- À Coordenação de Nível de Aperfeiçoamento de Pessoal de Nível Superior (CAPES), pela concessão de bolsa de doutorado e aos funcionários do Núcleo de Pesquisa para o Trópico Semiárido, pelo auxílio no manejo dos animais durante as contagens.

\section{REFERÊNCIAS}

Alves-Branco F.P.J., Pinheiro A.C. \& Sapper M.F.M. 1997. Epidemiologia da Haematobia irritans na região da campanha do RS: dados preliminares. Revta Brasil. Parasitol. Vet. 6(Supl. 1):46.

Barros A.T.M. 2001. Dynamics of Haematobia irritans irritans (Diptera: Muscidae) infestation on Nellore cattle in the Pantanal, Brazil. Mem. Inst. Oswaldo Cruz 96(4):445-450. <http://dx.doi.org/10.1590/S007402762001000400002><PMid:11391414>

Barros A.T.M., Saueressig T.M., Gomes A., Koller W.W., Furlong J., Girão E.S., Pinheiro A.C., Alves-Branco F.P.J., Sapper M.F.M., Braga R.M. \& Oliveira A.A. 2012. Susceptibility of the horn fly, Haematobia irritans irritans (Diptera: Muscidae), to insecticides in Brazil. Revta Bras. Parasitol. Vet. 21(2):125-132. <http://dx.doi.org/10.1590/S1984-29612012000200010> $<$ PMid:22832752>

Bianchin I. \& Alves R.G.O. 2002. Mosca-dos-chifres, Haematobia irritans: comportamento e danos em vacas e bezerros Nelore antes da desmama. Pesq. Vet. Bras. 22(3):109-113. <http://dx.doi.org/10.1590/S0100 736X2002000300004> 
Bianchin I., Koller W.W. \& Detmann E. 2006. Sazonalidade de Haematobia irritans no Brasil Central. Pesq. Vet. Bras. 26(2):79-86. <http://dx.doi. org/10.1590/S0100-736X2006000200004>

Bianchin I., Koller W.W., Alves R.G.O. \& Detmann E. 2004. Efeito da moscados-chifres, Haematobia irritans (L.) (Diptera: Muscidae), no ganho de peso de bovinos Nelore. Ciência Rural 34(3):885-890. <http://dx.doi. org/10.1590/S0103-84782004000300035>

Castro E., Gil A., Piaggio J., Chifflet L., Farias N.A., Solari M.A. \& Moon R.D. 2008. Population dynamics of horn fly, Haematobia irritans irritans (L.) (Diptera: Muscidae), on Hereford cattle in Uruguay. Vet. Parasitol. 151(2/4):286-299. <http://dx.doi.org/10.1016/j.vetpar.2007.10.020><PMid:18093739>

Collares N.C. 1990. Alguns aspectos etológicos da Haematobia irritans em Roraima. Dissertação de Mestrado, Universidade Federal de Minas Gerais, Belo Horizonte, MG. 56p.

Christensen C.M. \& Dobson R.C. 1979. Effects of testosterone propionate on the sebaceous glands and subsequent attractiveness of Angus bulls and steers to horn flies, Haematobia irritans (Diptera: Muscidae). J. Kansas Ent. Soc. 52(2):386-391.

Cruz-Vázquez C., Hernandez J.B., Mendonza I.V., Parra M.R., Martinez M.T.Q. \& Vazquez Z.B. 2000. Distribución anual de Haematobia irritans (L.) (Diptera: Muscidae) en tres establos lecheros de Aguas Calientes, México. Vet. Mex. 31(3):195-199.

Dias N.L., Okawa C.M.P., Araújo A.A.M. \& Gobbi M.F. 1999. Estudo dos impactos sobre o clima regional do reservatório de Itaipu. Seminário Nacional de Produção e Transmissão de Energia Elétrica, 15, Foz do Iguaçu, PR, p.1-6.

Gordon D.V., Haufe W.O. \& Klein K.K. 1984. Determination of economic thresholds for horn fly control in Western Canada: A farm level simulation approach. Can. J. Agricult. Econ. 32(2):399-421. <http://dx.doi. org/10.1111/j.1744-7976.1984.tb02134.x>

Grisi L., Leite C.R., Martins S.R.J., Barros M.T.A., Andreotti R., Cançado D.H.P., León P.A.A., Pereira B.J. \& Villela S.H. 2014. Reassessment of the potential economic impact of cattle parasites in Brazil. Revta Bras. Parasitol. Vet. 23(2):150-156. <http://dx.doi.org/10.1590/S1984-29612014042> $<$ PMid:25054492>
Guglielmone A.A., Anziani O.S., Mangold A.J., Giorgi R.E., Volpogni M.M. \& Flores S.G. 1997. Seasonal variation of Haematobia irritans (Diptera: Muscidae) in a recently infested region of central Argentina. Bull. Entomol. Res. 87(01):55-59. <http://dx.doi.org/10.1017/S000748530003635X>

INMET 1993. Instituto Nacional de Meteorologia. Normais Climatológicas 1961-1993. Disponível em <www.inmet.gov.br> Acesso em 3 mar. 2015.

Lima L.G.F., Perri S.H.V. \& Prado A.P. 2003. Variation in population density of horn flies (Haematobia irritans irritans) (L.) (Diptera:Muscidae) in Nellore cattle (Bos indicus). Vet. Parasitol. 117(4):309-314. <http://dx.doi. org/10.1016/j.vetpar.2003.10.002 > <PMid:14637033>

Maciel W., Lopes W.D., Cruz B., Teixeira W., Felippelli G., Sakamoto C.A., Fávero F.C., Buzzulini C., Soares V., Gomes L.V., Bichuette M. \& da Costa A.J. 2015. Effects of Haematobia irritans infestation on weight gain of Nelore calves assessed with different antiparasitic treatment schemes. Prev. Vet. Med. 118(1):182-186.<http://dx.doi.org/10.1016/j.prevetmed.2014.11.006> <PMid:25465474>

Morrison D.G. \& Foil L.D. 1995. Effect of horn fly (Diptera: Muscidae) control during the spring on calf production by fall-calving beef cows. J. Econ. Entomol. 88(1):81-84. <http://dx.doi.org/10.1093/jee/88.1.81> $<$ PMid:7884078>

Oliveira G.P. \& Freitas A.R. 1997. Comportamento da Haematobia irritans em fazendas com diferentes manejos de bovinos. Ciência Rural 72(2):279-284. <http://dx.doi.org/10.1590/S0103-84781997000200018>

Santos Júnior R.R. \& Caracristi I. 2012. Produção de dados climáticos primários a partir de medições em campo: procedimentos para o estudo do clima na região do açude Castanhão, CE. Revta Geonorte, edição especial 2,1(5):740-753.

SAS Institute 1989. SAS/STAT User's Guide, version 6. 4th ed. SAS Institute, Cary, NC.

Souza A.P., Bellato V., Ramos C.I., Dalagnol C.A. \& Henschel G.S. 2005. Variação sazonal de Haematobia irritans no planalto catarinense e eficiência do “Controle Dirigido". Revta Bras. Parasitol. Vet. 14(1):11-15. 